\title{
Replacing failed composite resin veneers with ceramic veneers: a case report
}

\begin{abstract}
Recently, ceramic veneers have been considered as one of the treatment alternatives for patients with esthetic problems involving anterior teeth, due to their color stability and high resistance against abrasion, requiring minimal tooth preparation. The aim of this study is to report esthetic rehabilitation by replacing the composite resin veneers of the anterior teeth with ceramic veneers in a 40-year-old male patient, who visited the restorative dental clinic at King Saud University, Saudi Arabia. After the clinical examination, the primary impressions were obtained for constructing the diagnostic models. Desired shade for the veneers was selected and previous composite resin veneer restorations were removed and the maxillary anterior teeth were prepared to receive ceramic veneers. Final impression was obtained and temporization was performed. A week later, temporary restorations were removed; subsequently, final cementation of the ceramic veneers was done. Ceramic veneers provide a superior alternative to composite resin veneers for esthetic modification of teeth. They are considered to be a successful treatment option that helps preserve the tooth structure while providing excellent aesthetic results and patient satisfaction.
\end{abstract}

Volume 10 Issue 5 - 2019

\author{
Ahoud Alshamrani \\ Department of Restorative Dental Sciences, King Saud \\ University, Saudi Arabia
}

Correspondence: Ahoud Alshamrani, BDS, MSc, Lecturer, Department of Restorative Dental Sciences, King Saud University, Riyadh, Saudi Arabia, Email ahalshamrani@ksu.edu.sa

Received: October 01, 2019| Published: October 09, 2019

Keywords: ceramic veneers, dental esthetics, ceramic restoration, composite resin, esthetic rehabilitation

\section{Introduction}

Currently, esthetics is an important reason for a patient to seek dental treatment. Based on the previous reports, more than half of the patients are dissatisfied with the general appearance of their teeth. ${ }^{1,2}$ Several factors affect the dental esthetics, such as the tooth color, shape, quality of the restorations, and arrangement of the dentition, especially the anterior teeth. ${ }^{3}$ Unesthetic anterior teeth could be treated by tooth bleaching, microabrasion, and composite resin restorations or ceramic veneers, depending upon the severity of the case. The direct composite resin veneers are applied to the teeth in a single visit, cost less and easier to repair in comparison to the ceramic veneers. However, they are prone to staining and shrinkage that may cause postoperative complications such as microleakage. ${ }^{4}$ Although debonding, chipping of the ceramic veneers, and difficulty to repair are the major failures of ceramic veneers, ${ }^{5,6}$ they are considered to be one of the most common treatment options; this is due to their excellent esthetics, smooth surface finish, and the stability of the color and contour of the restoration. ${ }^{7,8}$ Moreover, they are known to provide predictable, stable, and satisfactory results for both the patient and the dentist. ${ }^{9}$

\section{Case presentation}

A 40-year-old male patient visited the restorative dental clinic at King Saud University, Saudi Arabia, with a complaint of unesthetic and defective composite resin veneers. A year ago, composite resin veneer restorations were performed for the patient's maxillary anterior teeth at a private clinic. The patient was not happy with the outcome and wanted to get the composite resin veneer restorations replaced with highly esthetic restorations.

\section{Clinical examination}

The clinical examination revealed generalized staining of the teeth with defective composite resin veneers on the maxillary anterior teeth and spacing between the maxillary lateral incisors and canines, bilaterally (Figure 1). Also, the patient had a large amalgam restoration with inadequate root canal treatment of the maxillary left first premolar (Figure 2).

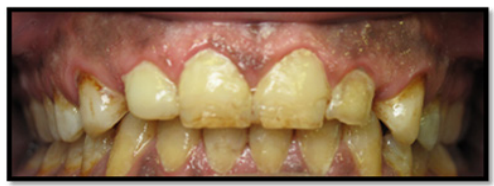

(a)

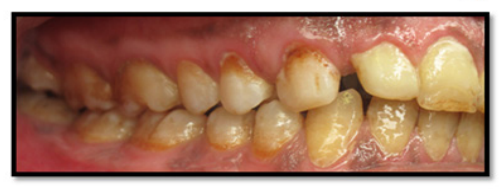

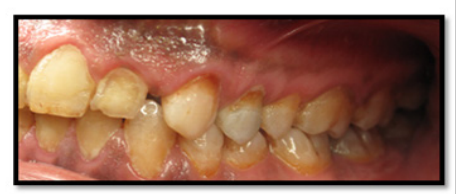

(c)

Figure I Preoperative clinical photographs: (a) frontal view, (b) lateral view: right side, and (c) lateral view: left side. 


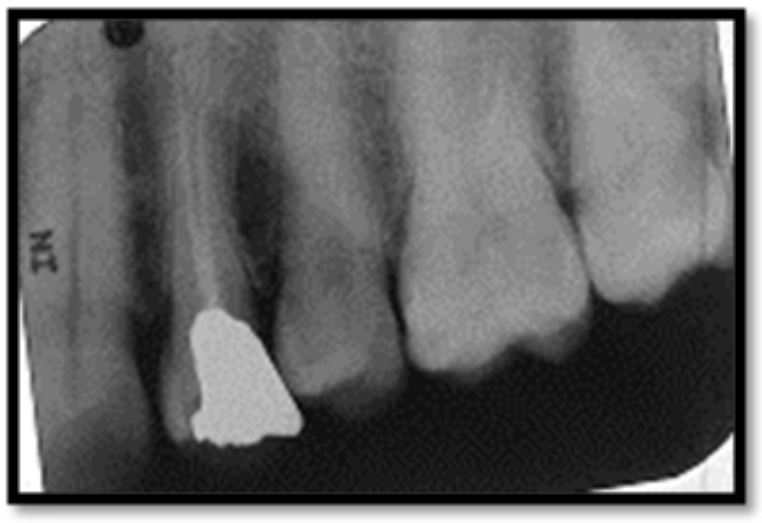

Figure 2 Periapical radiograph of maxillary left first premolar.

\section{Treatment plan}

After the clinical examination, radiographs and preoperative photographs were taken for the patient. Maxillary and mandibular alginate impressions were done for constructing the diagnostic models using type IV dental stone and mounted on a semi-adjustable articulator. Treatment options were discussed with the patient and he agreed for a ceramic crown restoration of the maxillary left first premolar and ceramic veneers for maxillary teeth, from the maxillary right second premolar to the maxillary left second premolar, for optimum esthetics and a better prognosis. Further, vital bleaching was suggested for the mandibular teeth due to their limited visibility. The patient was referred to an endodontic clinic for the re-treatment of maxillary left first premolar, which presented with an inadequate root canal treatment. Also, the defective composite resin veneers in the maxillary teeth were repaired temporarily using direct composite resin (IPS Empress Direct, Ivoclar Vivadent, Schaan, Liechtenstein) (Figure 3). During the following appointment, a diagnostic wax-up was presented to the patient and any desired alteration by the patient was analyzed, discussed, and adjusted. Also, the diagnostic models were used for the fabrication of a clear matrix for temporary restorations.
At the same visit, in-office bleaching was done for the patient using $35 \%$ hydrogen peroxide (Zoom! Whitespeed, Philips-USA) followed by an at-home bleaching procedure, using 15\% carbamide peroxide (opalescence PF 15\%, Ultradent Products, Inc., South Jordan UT, USA) with bleaching trays.

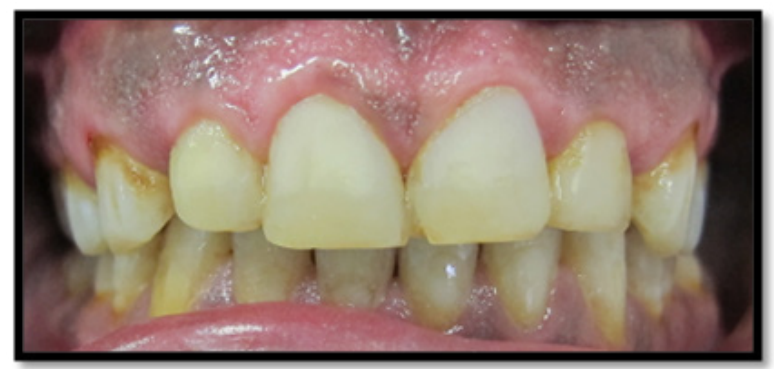

Figure 3 Repairing the defective composite resin veneers with composite resin restorations.

\section{Tooth preparation}

After two weeks, the patient was evaluated for shade changes and the desired shade for the veneers was selected using the VITAPAN classical shade guide (VITA Zahnfabrik, Germany). The composite resin veneers were removed and the maxillary anterior teeth were prepared to receive ceramic veneers using a flat-end tapered diamond bur. A depth of $0.5-0.75 \mathrm{~mm}$ was achieved for the facial reduction and $1.5 \mathrm{~mm}$ for the incisal reduction. A chamfer finish line was maintained at the level of the gingival margin. The proximal margin was extended into the facial and gingival embrasures. Following root canal treatment of the maxillary left first premolar, the tooth received a fiber post (FRC Postec Plus, Ivoclar Vivadent) and core build-up with composite material (Multicore Self Curing, Ivoclar Vivadent). Next, ceramic crown preparation was prepared using a cylindrical, tapered, round-end diamond bur with approximately $2 \mathrm{~mm}$ of axial and occlusal reduction. All the internal line angles were rounded and a definite shoulder finish line of $1 \mathrm{~mm}$ width was established all around the prepared tooth (Figure 4).

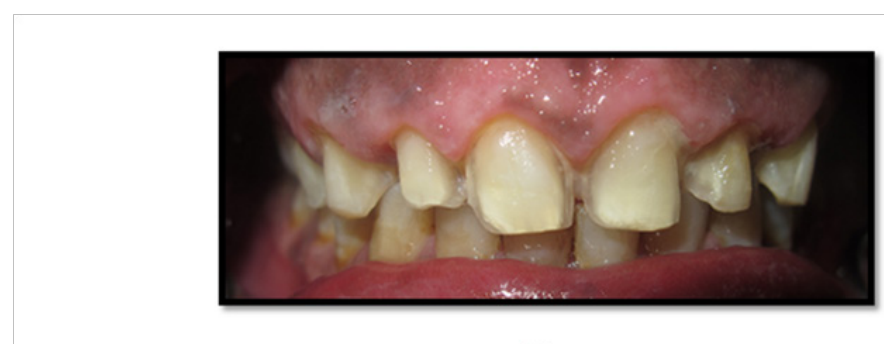

(a)

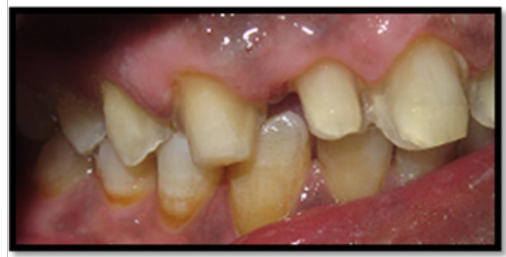

(b)

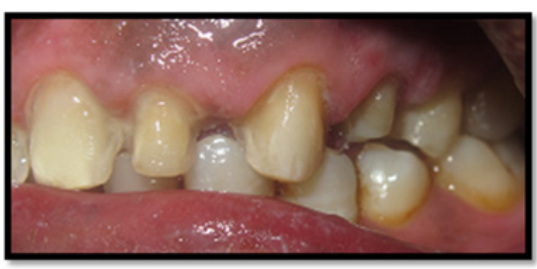

(c)

Figure 4 Maxillary teeth preparation: (a) frontal view, (b) lateral view: right side, and (c) lateral view: left side. 


\section{Final impression and temporization}

Following tooth preparation, gingival retraction was achieved using retraction cords (Ultrapak Cord \#00, Ultradent Products Inc., South Jordan, UT, USA) and hemostatic agent. Final impressions were taken using a polyvinylsiloxane material (Virtual, Ivoclar Vivadent, Amherst, NY) according to the manufacturer's instructions. Temporization was performed by spot-etching over the facial surface of each prepared tooth with $37 \%$ phosphoric acid (Total Etch, Ivoclar Vivadent, Schaan, Liechtenstein). Next, bonding agent (OptiBond Solo Plus, Kerr, Orange, CA, USA) was applied on the enamel-etched spots and light cured for 20 seconds using a high-intensity lightemitting diode (LED) curing light (Elipar S10, 3M ESPE, MN, USA). The temporary material (Protemp Plus, 3M ESPE, MN, USA) was loaded in the clear matrix and placed on the teeth until the material set. The matrix was gently removed, and the restorations were finished using diamond burs and polished using polishing discs.

\section{Ceramic restoration try-in and cementation}

After one week, the temporary restorations were removed, and the teeth were cleaned. Ceramic veneers and crown were fabricated with a lithium disilicate-reinforced glass ceramic material (IPS e.max Press, Ivoclar Vivadent, Schaan, Liechtenstein). Ceramic veneers were tried-in using a transparent shade try-in paste (Variolink Veneer tryin paste, Ivoclar Vivadent, Schaan, Liechtenstein) to assess marginal adaptation and shade. Try-in of the crown was done before the final glazing. At this stage, occlusion, margins, and seating of the crown were evaluated. Next, the veneers were prepared for bonding. The internal surfaces of the ceramic restorations were etched according to the following steps: 1 st - etching with $5 \%$ hydrofluoric acid (IPS Ceramic Etching Gel) for 20 seconds; 2nd - water rinsing and air drying, 3rd - applying silane coupling agent (Monobond Plus, Ivoclar Vivadent, Schaan, Liechtenstein) and gently air-drying after a minute. Later, the prepared teeth they were etched using $37 \%$ phosphoric acid for 30 seconds, rinsed, and dried. A clear mylar strip was placed interproximally to prevent bonding to the adjacent tooth. For the veneers prepared teeth, a layer of bonding agent (Adhese Universal, Ivoclar Vivadent, Schaan, Liechtenstein) was applied on the prepared tooth surfaces and air-thinned. Next, Heliobond (Ivoclar Vivadent, Schaan, Liechtenstein) was applied over the prepared tooth surfaces. The inner surface of the veneers was covered with light-cured resin cement (Variolink Veneer, transparent shade, Ivoclar Vivadent, Schaan, Liechtenstein). Veneers were positioned appropriately on the teeth by applying gentle pressure, followed by carefully removing the excess resin cement with an explorer before light curing. Initially, the light curing was performed for 2 seconds, and the excess resin cement was removed with a microbrush. Subsequently, each veneer was lightcured from the facial and lingual surfaces for 40 seconds. First, the two veneers of the central incisors were cemented simultaneously, followed by the cementation of veneers of the two lateral incisors. Next, the veneers of the two canines were cemented. Later, the Syntac Primer (Ivoclar Vivadent, Schaan, Liechtenstein) was placed in the crown preparation for 15 seconds and gently air-dried. This was followed by the application of Syntac Adhesive (Ivoclar Vivadent, Schaan, Liechtenstein) for 10 seconds and air-drying. Subsequently, Heliobond was applied over the tooth preparation. Finally, the dual-cure resin cement (Variolink N, Ivoclar Vivadent, Schaan, Liechtenstein) was placed over the inner surface of the crown and cemented over the prepared tooth. Light curing was done from the facial and lingual surfaces for 20 seconds. All the excess luting cement was removed with a number 12 surgical blade and explorer. Flossing was performed at the proximal areas to ensure interproximalcontact patency. A flame-shaped fine diamond bur was used to finish the ceramic margins. The margins were polished with a silicone instrument Pogo ${ }^{\circledR}$ (Dentsply/Caulk, Milford, DE, USA). Finally, the restorations were checked for any occlusal interference, thereby completing the final restoration procedure (Figure 5).

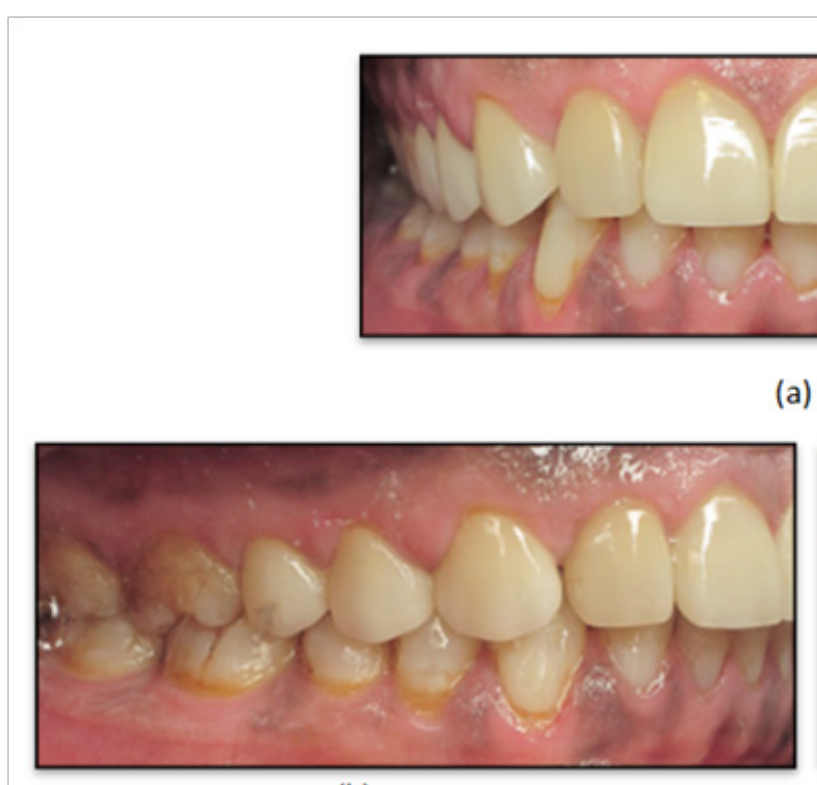

(b)

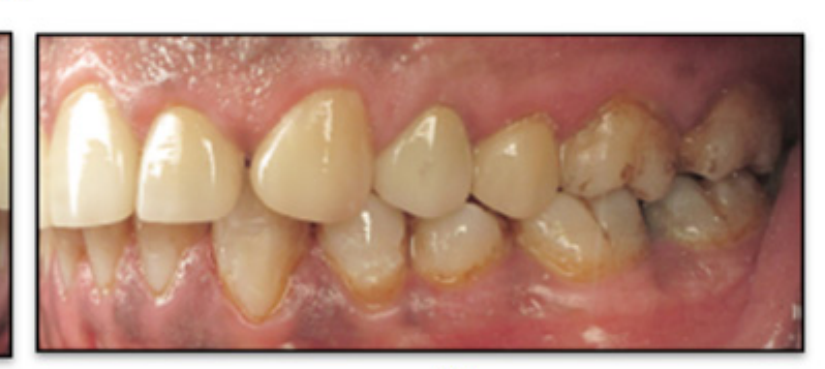

(c)

Figure 5 Postoperative clinical photographs: (a) frontal view, (b) lateral view: right side, and (c) lateral view: left side. 


\section{Discussion}

Since the introduction of composite resins, they have gained popularity as restorative materials because they require minimal tooth reduction and provide high esthetics. ${ }^{10}$ Currently, composite resin is the first choice material for restoring teeth and the direct composite resin veneer is an effective treatment option for treating unesthetic anterior teeth. Composite resins have several advantages over ceramic veneers; they are applied directly over the prepared tooth surfaces at the same visit, require minimal tooth preparation, and are an economical and aesthetic option. ${ }^{11}$ However, marginal leakage, susceptibility to discoloration and low wear resistance may affect the long-term esthetic results. ${ }^{12,13}$ Ceramic veneers, on the other hand, have increasing popularity over direct composite resin veneers as they provide more predictable and esthetic results with a higher success rate over a period of seven years. ${ }^{10,14}$ Also, the surface changes, including defects, staining at margins, and slight roughening of the surface, occur more frequently in composite resins than in ceramic veneers. ${ }^{15}$ Therefore, ceramic veneers were chosen to replace the existing composite resin veneers in this case. The ceramic veneer restorations provide an esthetic conservative treatment option with minimal reduction of the tooth structure. ${ }^{16}$ Although debonding is one of most common failures of ceramic veneers, which results due to the lack of adequate enamel for bonding, ${ }^{17}$ previous reports found that the survival rate is $78 \%$ for direct composite resin veneers and $94 \%$ for ceramic veneers after 2.5 years. ${ }^{18}$ Furthermore, ceramic veneers are known to provide significantly greater patient satisfaction than resin composite veneers. ${ }^{18}$

\section{Conclusion}

The demand for dental esthetics is steadily growing. Ceramic veneers provide a superior alternative to composite resin veneers for esthetic modification of teeth. They are considered to be a successful treatment option that helps preserve the tooth structure while providing excellent aesthetic results and patient satisfaction.

\section{Funding details}

None.

\section{Acknowledgements}

None.

\section{Conflicts of interest}

The author declares that there is no conflict of interest.

\section{References}

1. Enabulele J. Omo J. Self-perceived satisfaction with dental appearance and desired treatment to improve aesthetics. African Journal of Oral Health. 2017;7(1):7.

2. Akarslan ZZ. Dental esthetic satisfaction, received and desired dental treatments for improvement of esthetics. Indian J Dent Res. 2009;20(2):195-200.

3. Tin OMM, Saddki N, Hassan N. Factors influencing patient satisfaction with dental appearance and treatments they desire to improve aesthetics. BMC Oral Health. 2011;11:6.
4. Cramer NB, Stansbury JW, Bowman CN. Recent advances and developments in composite dental restorative materials. J Dent Res. 2011;90(4):402-416.

5. Peumans M. A prospective ten-year clinical trial of porcelain veneers. $J$ Adhes Dent. 2004;6(1):65-76.

6. Burke FJ. Survival rates for porcelain laminate veneers with special reference to the effect of preparation in dentin: a literature review. $J$ Esthet Restor Dent. 2012;24(4):257-265.

7. Farronato D. Esthetic integration between ceramic veneers and composite restorations: a case report. Annali di stomatologia. 2012;3(34).

8. Rotoli BT. Porcelain veneers as an alternative for esthetic treatment: clinical report. Oper Dent. 2013;38(5):459-466.

9. Radz GM. Minimum thickness anterior porcelain restorations. Dent Clin North Am. 2011;55(2):353-370.

10. Monaraks RC, Leevailoj. The Longevity of Ceramic Veneers: Clinical Evaluation of Mechanical, Biologic and Aesthetic Performances of Ceramic Veneers, a 7-year Retrospective Study. The Journal of the Dental Association of Thailand. 2018;68(3):288-301.

11. Korkut B, Yanikoglu F, Gunday M. Direct composite laminate veneers: three case reports. J Dent Res Dent Clin Dent Prospects. 2013;7(2):105-111.

12. Alonso V M, Caserio. A clinical study of direct composite full-coverage crowns: long-term results. Oper Dent. 2012;37(4):432-441.

13. Demarco FF. Anterior composite restorations: A systematic review on long-term survival and reasons for failure. Dent Mater. 2015;31(10):1214-1224

14. Granell-Ruiz M. A clinical longitudinal study 323 porcelain laminate veneers. Period of study from 3 to 11 years. Med Oral Patol Oral Cir Bucal. 2010;15(3):e531-e537.

15. Gresnigt MM, Kalk W, Ozcan M. Randomized clinical trial of indirect resin composite and ceramic veneers: up to 3-year follow-up. $J$ Adhes Dent. 2013;15(2):181-190.

16. Shetty A. Survival rates of porcelain laminate restoration based on different incisal preparation designs: An analysis. J Conserv Dent. 2011;14(1):10-15

17. Friedman MJ. Porcelain veneer restorations: a clinician's opinion about a disturbing trend. J Esthet Restor Dent. 2001;13(5):318-327.

18. Meijering AC. patients' satisfaction with different types of veneer restorations. J Dent. 1997;25(6):493-497. 